\title{
Mobile Data Management Structure Design based on Mobile Database
}

\author{
Xin $\mathrm{Hu}$ \\ Library, Nanchang Institute of Technology, Jiang Xi 330013, China \\ huxii@163.com
}

Keywords: Mobile database, data management, mobile computing, structure design.

\begin{abstract}
The mobile database is using SQL Server CE mobile database engine. Data conversion layer provides backup restore function (fully backup and restore, table with import and export) of SQL Server CE' local database, and to provide similar interface functions of storage procedure (it include: function of operating local database, function of remote upload and download, function of operation remote database) for upper mobile applications. Application is running on a mobile device and need to be provided services by the mobile database.
\end{abstract}

\section{Introduction}

In recent years, with the graphic development of network technology and wireless communication technology, people's target is hope to achieve unconstrained communication and sharing resources, this is a flexible and complicated distributed computing environment and usually called mobile computing environment. The main characteristics of mobile computing environment is the mobility, frequent broken, diversification network condition, and these characteristics make mobile computing environment can not be supported by the traditional distributed database technology effectively, and formed a kind of new database technology, i.e. the mobile database [1].

Microsoft SQL Server Windows CE Edition (SQL Server CE) is a streamlining database product launched by Microsoft and is suitable for mobile application development, with more stable function and performance. But there are some deficiencies when SQL Server CE will be applied to some mobile applications SQL Server CE does not have local backup and restore function, and SQL Server CE can not provides a stored procedure for application. In order to remedy the deficiency of SQL Server CE, the document proposed that a mobile database management model for mobile applications. And this model is divided into three layers structure: mobile database, data conversion, applications. [2]

At present, there are relatively perfect mobile database products: product family of Sybase SQL Anywhere studio [3], Microsoft product family in the Microsoft SQL Server Windows CE edition, the National People's Congress of small Jin Ling mobile database.

For now, SQL Server Windows CE Edition Microsoft can be said to be a more comprehensive and stable performance of mobile database. However, Microsoft SQL server, Windows CE edition in the specific application interface function and have some inconvenience, for example, do not have a local backup and recovery and function of import and export, and did not application with stored procedures.

\section{Organization of the Text}

This paper mainly discusses the in mobile computing environment, mobile database data management technology, the basic theory and implementation methods, and according to the traditional database management technology, for mobile electronic commerce application, design a reasonable mobile database data management model. The main research contents include:

(1) Study the characteristics of the mobile computing environment, according to the characteristics of the data management strategy. 
(2) Research on the differences and similarities between the traditional database data management and mobile database data management.

(3) Research on the characteristics of mobile e-commerce, according to the characteristics of the design of a reasonable data management model of mobile database.

(4) Research on the functions and features of SQL Server Windows CE Edition Microsoft mobile database.

(5) According to the data management model of Server Windows CE Edition Microsoft mobile database, the function of SQL is improved.

(6) Data management model for mobile database.

(7) The design and implementation of a SQL Server Windows CE Edition Microsoft mobile database data management tool, in order to test the various functional modules. [4]

Table 1 SQL Server CE

\begin{tabular}{cc}
\hline SQL Server Type & SQL Server CE \\
\hline bigint & bigint \\
datetime & datetime \\
float & float \\
binary & binary \\
bit & bit \\
image & image \\
character & National character or ntext \\
money & money \\
decimal & numeric \\
real & real \\
Double precision & Double precision \\
integer & integer \\
National character varying (max) & ntext \\
ntext & ntext \\
smalldatetime & datetime \\
Smallint (int 2) & Smallint (int 2) \\
Sql_variant & RDA \\
text & ntext \\
numeric & numeric \\
uniqueidentifier & uniqueidentifier \\
Varbinary (n) & Varbinary (n) \\
\hline
\end{tabular}

\section{Literature References}

As the extension and expanding of the distributed database, mobile database, which has many excellences of distributed database and unique character itself, can satisfy the need that people want to get any information in any time wherever they are, therefore it has quite extensive foreground in application. In the mobile database system, [5] because of the properties of the mobile environment such as mobility, frequent disconnection, network diversity, limited power .etc, the technique of the traditional distributed database can't sustain effectively the mobile environment; hence the research for the technique in the mobile environment is to be essential.

Table 2 The compare of synchronous replication technology in primary mobile database product

\begin{tabular}{cccc}
\hline name & $\begin{array}{c}\text { Sybase SQL } \\
\text { Anywhere }\end{array}$ & Xiao jinling & Openbase \\
\hline 1 & Windows CE; & Windows CE; & Windows CE; \\
& Palm OS & Hopen; & Plam OS; \\
& Linux & Hopen \\
2 & MobiLink & Sync Server & $\begin{array}{c}\text { Open Base Mini } \\
\text { Server }\end{array}$ \\
3 & ODBC & ODBC & ODBC; JDBC \\
\hline
\end{tabular}


Shown in Table 1 and2; Based on the domestic and international information, this paper mainly studies the replication technique and cache technique of the mobile database and has done the work as follows:

1) About the replication technique, based on the two-tier-replication, this paper introduces three-tier-replication and then proposes the periodical incremental maintenance algorithms with regard to the third tier replication (mobile client cache). Furthermore, it analyses the reason why three-tier-replication technique sustains effectively the mobile environment. 2) About the cache technique, firstly this paper elaborates the reason why the traditional cache technique can't be effective in the mobile database and analyses the priority-based incremental update algorithm. Furthermore, one dynamic priority-based incremental update algorithms(DPIU) which considers two factors of data complexity and user's access probability is proposed The DPIU which inherits the concept of data fresh and adds factors such as data complexity, makes up the shortages of the priority-based incremental update algorithm. Hence, the algorithm improves effectively the real-time and consistency in view update of mobile database, meanwhile caters to the demand of the mobile users. (Fig. 1)

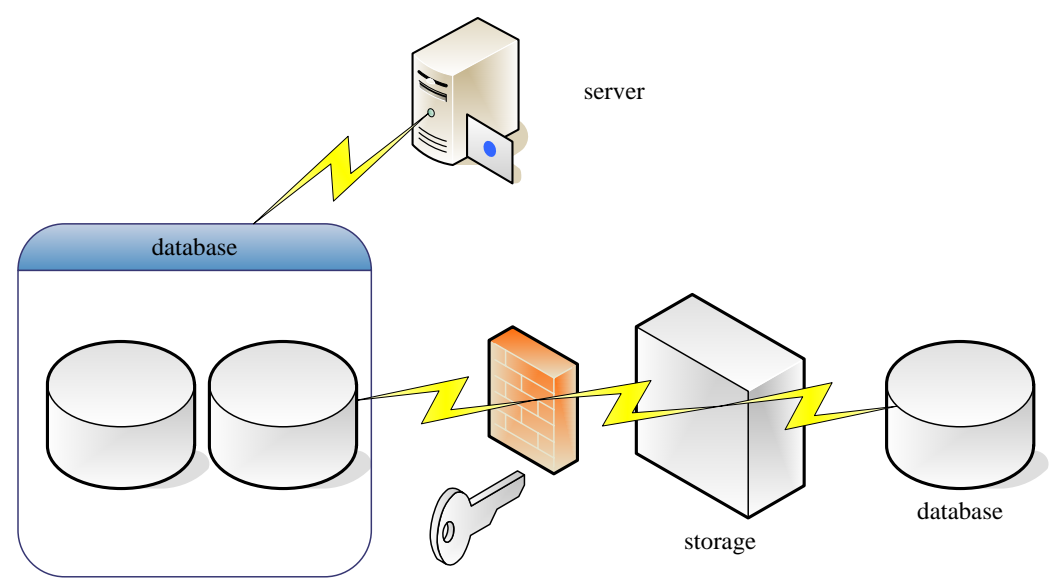

Fig. 1 Database Application Model

\section{Key technology}

Because the wireless mobile communication network is unbalanced and requires good scalability of users, it's suitable to transfer information with the data broadcast method in mobile computing environment. In order to reduce tuning time in the mobile clients, indexes can be added into the broadcast. But the addition of index increases the length of the broadcast message, thus increases the access time of mobile clients. People have made a lot research and put forward many methods of broadcast tuning on reducing accessing time. However, how to optimize both tuning time and accessing time of mobile clients has become a tough researching topic on broadcasting technology. We post a policy that the mobile client establishes broadcast index locally, which can make both tuning time and accessing time in the mobile client optimized. (Eq. 1)

$$
\mathrm{P}=\left\{\begin{array}{lc}
0 & \Delta \mathrm{t} \leq T_{j} \\
w_{j} \Delta t & 0<\Delta \mathrm{t}<T_{j} \\
w_{j} & \Delta \mathrm{t} \geq T_{j}
\end{array}\right.
$$

After disconnection, mobile clients execute transactions locally based on the cached data. After reconnection, the transactions in the mobile clients will be submitted as the tentative transactions to the stable hosts to re-execute. Using this method, if there's a high probability of conflicts, the probability of successful submitting of the tentative transactions will be very low. Lots of abortion of tentative transactions will waste server and communication resources, reduce the performance of the whole system. In order to increase success probability for the re-submitting of tentative 
transactions, we provide a method to submit tentative transactions according to probability and transmit tentative transaction logs in groups. If used in the environments with high conflicting probability, this method can save the costs of transmission on mobile clients, reduce the load on the stable hosts and improve the number of transactions the system can process. (Eq. 2)

$$
f_{t}(\Delta t)=\left\{\begin{array}{lr}
1-\Delta t / T & 0 \leq \Delta t<T \\
0 & \Delta t \geq T
\end{array}\right.
$$

In order to improve average responding time, reduce the usage of network bandwidth and improve data availability, mobile clients usually cache part of the data locally. How to keep cache consistent is an important study topic. We use the method based on broadcasting cache invalidating report from the servers to keep cache consistent. We study the mobile client cache consistency in the following three prospects: (Eq. 3)

$$
\overline{w_{j}}=\frac{\frac{w_{j} p_{i j}}{o\left(s_{i j}\right)}}{\sum_{j=1}^{n} \frac{w_{j} p_{i j}}{o\left(s_{i j}\right)}}
$$

Many paper have posted the method on periodically broadcasting cache-invalidating reports and promptly broadcasting cache-invalidating reports. We provide a method on broadcasting cache-invalidating reports with variant periods and analyzes its performance and availability. (Eq. 4)

$$
F_{s u m}=\frac{\sum_{i, j \in \Omega} \frac{w_{i} p_{i j}}{o\left(s_{i j}\right)} f_{i}\left(V t_{i j}\right)+\sum_{i, j \in \Omega} \frac{w_{i} p_{i j}}{o\left(s_{i j}\right)}}{\sum_{i=1}^{m} \sum_{j=1}^{n} \frac{w_{j} p_{i j}}{o\left(s_{i j}\right)}}
$$

To support offline operations, the invalidating report IR, broadcast from the fixed server at time T; has to include data collection of invalidating report periods before $\mathrm{T}$ is called invalidating report time window. To use the method to effectively keep cache consistent while the mobile clients disconnect and reconnect frequently, the key point is to select proper value of $\}$.We provide a method to dynamically adjusting invalidating report window. It theoretically deduces the computing equation for analyzes the proper value and implements the method. (Eq. 5)

$$
V F=F_{\text {sum }}-F(V)=\frac{\sum_{i, j \in \Omega} \frac{w_{j} p_{i j}}{o\left(s_{i j}\right)}-\sum_{i, j \in \Omega} \frac{w_{j} p_{i j}}{o\left(s_{i j}\right)} f_{j}\left(V t_{i j}\right)}{\sum_{i=1}^{m} \sum_{j=1}^{n} \frac{w_{j} p_{i j}}{o\left(s_{i j}\right)}}=\frac{\sum_{i, j \in \Omega} \frac{w_{j} p_{i j}}{o\left(s_{i j}\right)}\left(1-f_{j}\left(V t_{i j}\right)\right)}{\sum_{i=1}^{m} \sum_{j=1}^{n} \frac{w_{j} p_{i j}}{o\left(s_{i j}\right)}}
$$

If the disconnection time of the mobile client is longer than broadcasting periods, it can't use invalidating report to keep cache consistent. We provide an array validating method. Although the method often can only locate the range of error pages and can't find the exact page, it isn't the whole cache in the invalidated mobile client and it keeps most cache that's still valid, thus greatly reduced data transmission.

\section{Conclusions}

Mobile database technology is distributed database and distributed computing technology of the important branches. Mobile database is mobile computing environment in order to meet the data needs of access and development and produce usually can be seen as a traditional distributed database expansion. Mobile computing environment compared with traditional distributed computing 
environment with the new characteristics, traditional distributed database technology can not be fully applied to the mobile database. Based on the application of mobile computing devices has become increasingly popular. Widespread use of personal digital assistants (PDA), smart phones and other mobile handheld devices anytime, anywhere for business activities. Users will be applied data from the central database system downloaded to mobile handsets, and its off-line access and modify. In order to adapt to the development of mobile applications, a new technology-Mobile database technology came into being, and becomes a study of a hot issue.

\section{References}

[1] M. Satyanarayanan. Workshop on mobile computing systems and applications[J]. IEEE Personal communications, 2015, 2(2): 11-19.

[2] D. Barbara. Mobile computing and database-A survey[J]. IEEE Transactions on knowledge and data Engineering, 2009, 11(1): 108-117.

[3] G H. Forman and J. Zahorjan. The Challenges of Mobile Computing. IEEE Computer, 2004,17(4):38-47.

[4] O. Wolfson, S.Jajodia and YX.Huang. An Adaptive Data Replication Algorithm. ACM Transactions on Database Systems, June 2007, 22(2): 255-314.

[5] K.S. Lee and Y H. Chin. A New Replication Strategy for Unforeseeable Disconnection under Agent-Based Mobile Computing System. In: Proceedings of International Conference on Parallel and Distributed System,2008. 164-171. 